\title{
Mapping Control Survey in Urban Complex Environment Based on Multi-GNSS RTK Technology
}

\author{
Qiuying Guo*, Guangrong Hao and Wengang Sang \\ College of Surveying and Geo-informatics, Shandong Jianzhu University, Fengming Road, Jinan 250101, P.R. China \\ ${ }^{*}$ Corresponding author
}

\begin{abstract}
Mapping control survey for 13 control reference points were carried out based on RTK technology using GPS/BEIDOU/GLONASS multi-constellation compatible GNSS receivers in various urban environment (open, building shelter, tree shading, etc.). Operation scheme and technique flow were discussed. Operation efficiency and positioning accuracy in different environment were analyzed. Experimental results show that the horizontal position and elevation of the points measured by $R T K$ can reach $2 \mathrm{~cm}$ and $3 \mathrm{~cm}$ precision level respectively in open environment, which can meet the accuracy requirements for mapping horizontal and vertical control survey. However in environment such as building shelter or tree shading, the horizontal position of the points measured by RTK can reach $2 \mathrm{~cm}$ precision level generally, while the elevation biases are more than $3 \mathrm{~cm}$ mostly, which is difficult to achieve the accuracy of elevation mapping control survey.
\end{abstract}

Keywords-Multi-GNSS; RTK; observation environment; point calibration; mapping control survey

\section{INTRODUCTION}

With the development of Global Navigation Satellite System(GNSS), the horizontal position and elevation of the points can be measured quickly by RTK technology with 2$3 \mathrm{~cm}$ precision level. Especially in recent years, the development of multi-GNSS provides more favorable conditions. GNSS will enter a new situation with the constellation more than 100 satellites coexist with the development and improvement of multi-GNSS, especially rapid development of BEIDOU, which will play an important role in improving GNSS application ability in urban complex environment (such as high building, tree shanding, glass wall, viaduct). In order to test the performance of multi-GNSS RTK in urban region, mapping control survey experiments were carried out in university campus using GPS/BEIDOU/GLONASS multi-constellation compatible GNSS receivers in various environment. Operation scheme and technique flow were discussed. Operation efficiency and positioning accuracy in different environment were analyzed.

\section{EXPERIMENT ENVIORONMENT}

Representative experimental area was selected around the office building and sides of road. The experimental time period is May $10^{\text {th }}$-May 20th. There are 13 traverse control points(A1-A13) and one GPS control point(GPS6) in this area.

The precise coordinates and elevations of these points are known and regarded as reference values. These control points are distributed on both sides of the road, among which five control points are close to the building. There are trees on both sides of the road and there are dense canopies of some trees. Figure 1 gives the measurement environment at each point.

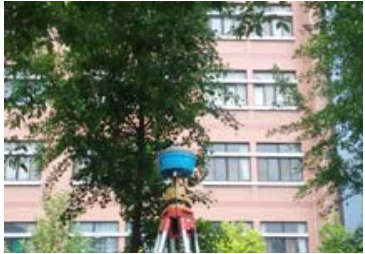

A1

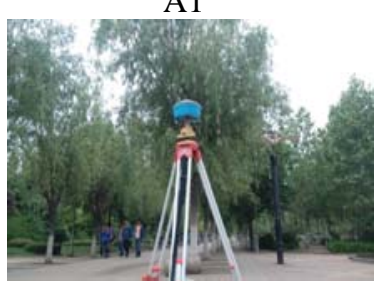

A3

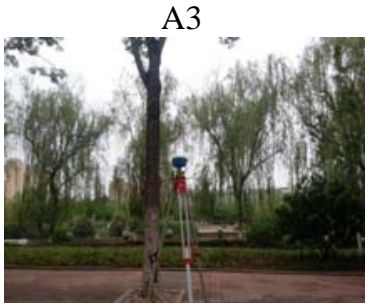

A5

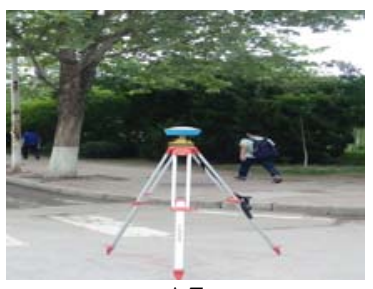

A7

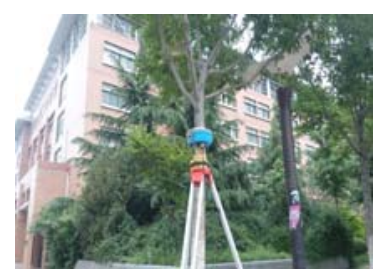

A2

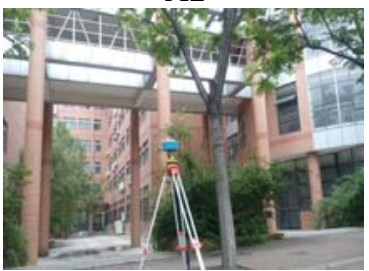

A4

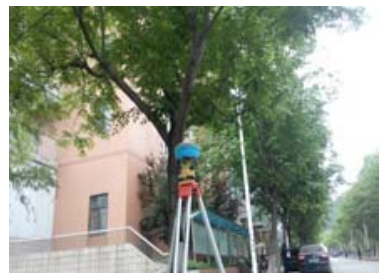

A6

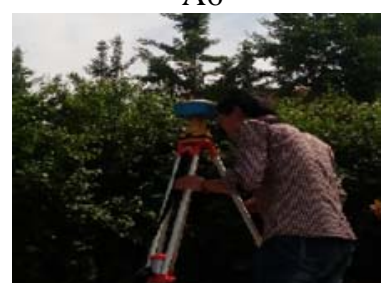

A8 


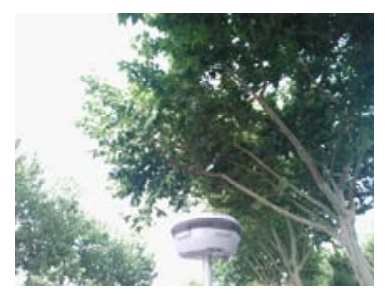

A9

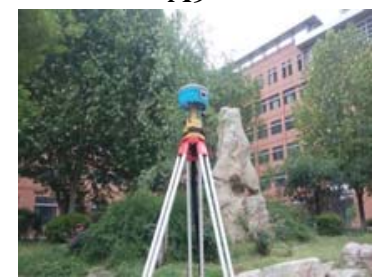

A11

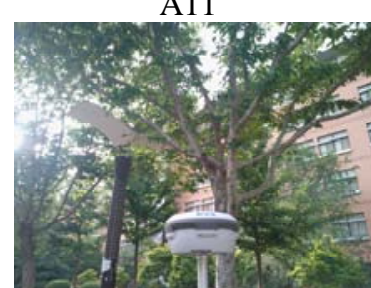

A13

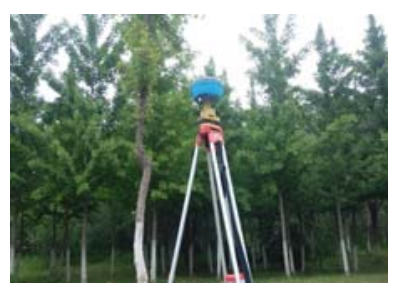

A10

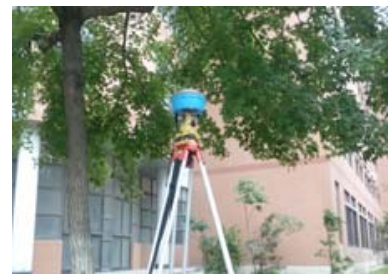

A12

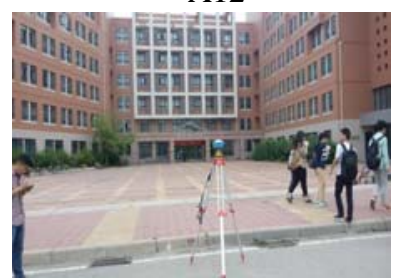

GPS6
FIGURE I. MEASUREMENT ENVIRONMENT FOR A1-A13 AND GPS6 CONTROL POINTS

Figure 1 shows that most of the 14 control points for experiments are located under occlusion environment. Among them, A1 and A2 locate about 6 meters south of the Civil Engineering office and there are shades above the two points. A4, A5 and A6 located on the west side of the Civil Engineering office building about 4 meters and there are dense leaves over the three points. A8 and A9 are far away from the building but there are trees near the two points. A11, A12 and A13 locate on the east side of the Science Building and close to the building, and there are also trees sheltered. There is no tree shelter above the point GPS6 but close to the Yifu Building. In contrast, observation conditions of A3, A7 and A10 are better than above-mentioned points, with wide vision, far away from buildings and no dense shades.

\section{EXPERIMENT AND RESULT ANALYSIS}

According to the distribution of experimental points and observation environment, we chose A3, A7 and A10 as the three known points for point calibration. The coordinate transformation parameters obtained in this way will meet the needs of the whole area. We used the three points to carry out point calibration in the morning, noon and afternoon periods, respectively. We used GPS6 as checkpoint. Most of the differences between the coordinates and elevations of the checkpoint and their known values are about $1 \mathrm{~cm}$, which meets the requirements of RTK measurement specification.
The instruments used in the experiment is three constellation compatible receivers of GPS/BEIDOU/GLONASS. The satellite cut-off angle is set to 15 degrees. The RTK measurements on each point were measured in three time periods. The first observation period is 8:00 9:00 am, the second observation period is 1:00 2:00 noon, and the third observation period is 6:00 7:00 pm. Then the coordinates and elevations of the control points measured by RTK are compared with the known coordinates and elevation values.

In order to ensure the accuracy and reliability of the mapping control points and combining RTK mapping control point requirements, we used the following experimental observation methods. Firstly, all control points of the RTK measurements are strict centering and leveling by three tripod legs. Secondly, all the observations are performed after the RTK fixed solution is stable and convergent, and "first fixed not recorded, second fixed recorded"are performed. Finally, 2 round observations were measured at each point, and each round collected 5 epochs. The average values of 2 rounds are taken as the final results of observation points.

The GPS/BEIDOU/GLONASS compatible receivers can receive about 20 satellite signals. PDOP values were generally less than 2. Experimental results of the A1-A13 control points measured by Multi-GNSS RTK tchnology are compared with their reference values. The differences between the RTK measured values and the reference values are shown in Table 1.

From Table 1, we can see that: The differences between RTK measurements of A3, A7, A10 stations and their reference values are smaller than those of the other stations. The differences of plane coordinates are generally less than $2 \mathrm{~cm}$ and the differences of heights are generally less than $3 \mathrm{~cm}$. While the differences between RTK measurements of A1, A2, A8, A9, A11and A13 stations and their reference values are slightly larger than that of A3, A7, A10 stations. But most of the differences of plane coordinates are generally less than $2 \mathrm{~cm}$, and most of the differences of heights are larger than $3 \mathrm{~cm}$. The differences of plane coordinates and heights are generally larger than $2 \mathrm{~cm}$ and $3 \mathrm{~cm}$ respectively for stations of A4, A5, A6 and A12, which are close to building.

\section{SUMMARY}

The experimental results of RTK measurement in various urban environment based on Multi-GNSS RTK technology showed that: point positions and elevations measured by RTK can meet the mapping plane and elevation control measurement precision under open environment. While plane positions under the environment of campus buildings and trees can generally satisfy the accuracy requirement of mapping plane control survey, and the height deviation of the measured points in the occlusion environment is generally larger than $3 \mathrm{~cm}$, which is difficult to achieve the accuracy of elevation control survey mapping. 
TABLE I. THE DIFFERENCES BETWEEN THE RTK MEASURED VALUES AND THE REFERENCE VALUES (CM)

\begin{tabular}{llllllllll}
\hline \multirow{2}{*}{ Point No. } & \multicolumn{3}{c}{ First observation period } & \multicolumn{3}{c}{ Second observation period } & \multicolumn{3}{c}{ Third observation period } \\
& $\mathbf{d x}$ & $\mathbf{d y}$ & $\mathbf{d H}$ & $\mathbf{d x}$ & $\mathbf{d y}$ & $\mathbf{d H}$ & $\mathbf{d x}$ & $\mathbf{d y}$ & $\mathbf{d H}$ \\
\hline A1 & 0.2 & 0.8 & 1.0 & 4.5 & 0.1 & 0.5 & 1.7 & 0.8 & 0.1 \\
A2 & 0.7 & 0.4 & 6.1 & 2.0 & 0.4 & 4.0 & 0.2 & 1.0 & 2.0 \\
A3 & 0.3 & 0.1 & 2.7 & 0.5 & 0.6 & 3.4 & 0.2 & 0.7 & 0.4 \\
A4 & 1.7 & 4.5 & 2.6 & 3.2 & 1.5 & 1.1 & 1.7 & 3.6 & 1.5 \\
A5 & 0.9 & 1.9 & 5.3 & 2.2 & 2.5 & 0.6 & 1.9 & 2.7 & 4.2 \\
A6 & 2.8 & 0.0 & 0.5 & 1.4 & 2.2 & 5.0 & 1.4 & 0.9 & 4.7 \\
A7 & 0.3 & 0.2 & 2.4 & 1.9 & 1.1 & 2.1 & 0.9 & 0.9 & 0.1 \\
A8 & 0.7 & 0.3 & 2.1 & 1.6 & 1.0 & 4.8 & 0.6 & 1.0 & 4.9 \\
A9 & 1.6 & 1.5 & 4.9 & 1.1 & 0.6 & 3.9 & 0.7 & 0.3 & 5.3 \\
A10 & 0.0 & 1.0 & 2.0 & 2.4 & 0.6 & 3.0 & 1.8 & 0.7 & 0.1 \\
A11 & 0.4 & 0.1 & 2.2 & 2.8 & 0.5 & 3.8 & 0.8 & 3.0 & 5.8 \\
A12 & 2.2 & 1.5 & 1.8 & 3.6 & 9.7 & 13.1 & 1.2 & 2.5 & 1.5 \\
A13 & 1.3 & 0.5 & 0.9 & 2.2 & 1.7 & 0.9 & 0.4 & 0.3 & 1.6 \\
\hline
\end{tabular}

\section{ACKNOWLEDGMENT}

This work is supported by the Technology Project Plan of Ministry of Housing and Urban-Rural Development of the People's Republic of China(2014-K8-070) and Shandong Provincial Department of Housing and Urban Rural Construction Technology Project Plan (2017-R1-004). The authors greatly appreciate the editor and the anonymous reviewers on this manuscript.

\section{REFERENCES}

[1] CH/T 2009-2010. Specifications for global positioning system real-time kinematic(RTK) surveys[S].

[2] Zhang He, Qian Linchun. The Application of GPS RTK in Mapping Control Survey[J]. Geomatics and Spatial Information Technology, 2007, 30(4): 59-61.

[3] Chen Junlin. Accuracy and Reliability Analysis on GPS-RTK in Traditional Control Surveying[J]. Geomatics and Spatial Information Technology, 2011, 34(5): 95-98.

[4] Li Zhiming. The application of Network RTK in Large Scale Mapping Control Survey[J]. Geospatial Information, 2012,10(3):134-135.

[5] Pan Chunjian, Jiang Yajun, etc.. Application of RTK Technique to Mapping Control Survey [J]. Survey and Mapping of Geology and Mineral Resources, 2012,23(1):30-32.

[6] Zhang Hengjing. Application of south single-base CORS in horizontal control network reconstruction in campus practice[J]. Journal of Liaoning Technical University (Natural Science), 2010, 29(z1): 38-39.

[7] A. Pirti, MA. Yucel, K. Gumus. Testing real time Kinematic GNSS (GPS and GPS/GLONASS) methods in obstructed and unobstructed sites[J]. Geodetski Vestnik, 2013, 57(3): 498-512. 